\title{
Notas para entender la migración: instituciones gubernamentales y política migratoria mexicanas en los albores del siglo XXI
}

\author{
RODOLFO CASILLAS R.*
}

RESUMEN: Durante la administración de Felipe Calderón los niveles de agresión y daño a los migrantes de paso, y también a migrantes mexicanos, llegaron a límites extremos con los secuestros y asesinatos masivos. La atención se centró en el crimen organizado, pero no se analizaron las insuficiencias, contradicciones y anacronismos de la ley, los problemas en su aplicación y los errores estratégicos en las medidas prácticas del Instituto Nacional de Migración de México. Sin analizar esos hechos, cualquier otra explicación sobredimensiona la parte delincuencial y subdimensiona lo que corresponde a lo jurídico y político. En este texto se presentan, en consecuencia, elementos de juicio sobre la actuación del Instituto, sus cuadros directivos y el mandato legal, en momentos en que el tema de la seguridad nacional subordinaba el tema migratorio. El resultado es una implosión institucional que evidencia, adicionalmente, el desfase entre realidad dinámica e instituciones anacrónicas y casi inmóviles.

PALABRAS CLAVE: migración de paso, seguridad nacional, marco legal, administración Calderón, prioridades gubernamentales.

* Profesor e investigador de la Facultad Latinoamericana de Ciencias Sociales (Flacso), México. 


\begin{abstract}
During the Calderón administration the amount of aggression and harm done to migrants in transit, and later also to Mexican migrants, reached extreme levels, with mass kidnappings and murders. The blame was placed squarely on organized crime, but the weaknesses, contradictions and anachronisms of the relevant laws, the difficulties in their application, and the strategic errors found in the actions of the National Migration Institute (INM) of Mexico went without scrutiny. Without an analysis of these facts, any explanation that went beyond laying the blame on the criminal element avoided the responsibility that fell to the legal and political systems. This article presents, then, a critical assessment on the activities of the INM, its management and mandate, in instances in which the topic of National Security overrode the topic of migration. The result is an institutional implosion that also reveals the discrepancy between a dynamic reality and outdated, almost immovable, institutions
\end{abstract}

KEY WORDS: transit migration, national security, legal frame, administración Calderón, government priorities, Calderon's administration 


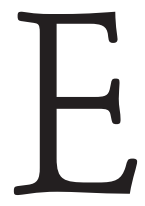

n el siglo XXI, los gobiernos mexicanos han tardado en contar con una agenda clara para atender la migración que transita por el país, pero hasta 2015 no lo han logrado. No hay elementos de juicio que permitan marcar un derrotero distinto al de subordinar ese flujo migratorio a prioridades nacionales distintas, como el combate al crimen organizado. Atender a quienes transitan sin documentos por México no ha sido un asunto de alta prioridad para la administración pública federal.

A México le urge una política migratoria de atención a los indocumentados, instituciones sólidas y burocracias profesionales que orienten las estructuras gubernamentales. La falta de estos componentes básicos ha permitido que desde 2007 el tráfico de personas terminara bajo el control de la delincuencia organizada. La descomposición de las instituciones y grupos organizados para controlar el tráfico resulta de la anacrónica agenda interna en materia migratoria. ¿̇Cómo se llegó a este nivel de gravedad, de participación polémica de la autoridad migratoria mexicana, de agresiones y daños inimaginables a los indocumentados, primero extranjeros y luego también mexicanos, y de consolidación de un nuevo nicho delictivo que hacía y hace del migrante su principal víctima?

Para dar elementos de respuesta es más que pertinente analizar la legislación mexicana vigente en materia migratoria, cómo se elaboran las políticas gubernamentales y establecen sus prioridades y cómo se nombran autoridades y las implicaciones de su nombramiento y actuación cuando su perfil no corresponde al mandato asignado. Para decirlo de manera llana: es necesario analizar el interior de las instituciones, no sólo las motivaciones de los migrantes, las instancias solidarias y las redes delictivas. Así sea de manera limitada y exploratoria, este texto propone ver esos interiores. Para ello, se recurre a fuentes orales que no son del todo identificadas, ${ }^{1}$ pues en su momento fueron funcionarios de mando medio superior a nivel central o superior en varias entidades federales, de instancias de gobierno federal en materias de seguridad, inteligencia y migración; de su testimonio y de su

${ }^{1}$ De esas personas sólo se anotan sus siglas para tranquilidad de quien legítimamente reclame saber la fuente de consulta, sin que se haga cita textual de sus palabras, pues no hubo permiso para grabar las entrevistas, mismas que se realizaron entre 2010 y 2011. 
cotejo con otros informantes, así como de consulta de material documental que se cita, se extraen los argumentos centrales que aquí se exponen.

\section{Antecedentes}

En la administración pública federal no ha existido una responsabilidad clara sobre la definición de estrategias ${ }^{2}$ para las instancias que administran los flujos migratorios indocumentados, ${ }^{3}$ la Policía Federal coadyuvante, el órgano de inteligencia y el Ministerio Público (MP) que investiga los ilícitos. Esta ausencia no había sido tan grave en el siglo xx, pero a partir de que se iniciaron los secuestros exprés de migrantes, en las postrimerías de la presidencia de Vicente Fox, la necesidad de subsumir la migración se volvió una urgencia social, pero inercias institucionales y otro tipo de prioridades impidieron la corrección, como se verá a continuación.

En la década de los noventa, el tema migratorio adquirió algún grado de importancia con la creación del Instituto Nacional de Migración (INM), el 9 de junio de 1993, con objeto de planear y administrar los servicios migratorios en la lógica de la integración de México al bloque económico del Tratado de Libre Comercio de América del Norte (TLCAN) que entró en vigor en 1994. Parte de lo novedoso de esta institución fue la creación de dos áreas sustantivas para la atención de la migración: la Coordinación de Regulación Migratoria, enfocada al creciente volumen de los flujos que empezaban a ingresar al país de manera documentada, ${ }^{4}$ y la Coordinación de Inspección

${ }^{2}$ Más allá de lo que digan las disposiciones legales, en los hechos cada dependencia trabaja generalmente de manera aislada, siempre bajo el prurito de la desconfianza mutua.

${ }^{3}$ Es pertinente recordar que hasta el 18 de mayo de 2005 el inm fue una entidad administrativa más, un órgano desconcentrado de la Secretaría de Gobernación, que no pertenecía al gabinete de seguridad; mas, como se vería luego, su adscripción a dicho gabinete sólo serviría para securitizar la migración; es decir, subordinar la atención de los flujos migratorios a la alta prioridad de atacar los cárteles de drogas ilícitas.

${ }^{4}$ Como resultado de la promoción turística, el crecimiento económico y el asentamiento de diversas empresas transnacionales.

$50 \frac{\text { PRIMER SEMESTRE } 2015}{\text { MIGRACIÓN Y DESARROLLO NÚM. } 24}$ 
y Asuntos Jurídicos, ${ }^{5}$ que en 1998 se convertiría en la Coordinación de Control y Verificación Migratoria, cuya finalidad es administrar los flujos indocumentados. Asimismo, se estableció un despliegue territorial más amplio a través de sus delegaciones regionales ${ }^{6}$ que, ya para 2000 , se habían transformado en delegaciones estatales, es decir, se instaló una delegación en cada estado del país. En las postrimerías de la presidencia de Felipe Calderón, habría una nueva modificación: las coordinaciones pasaron a llamarse direcciones generales. Pero la eventual trascendencia de esos cambios, junto a la vigencia de la Ley de Migración, expedida en 2011, marcan una temática para otro análisis.

Pese a que su nueva estructura lo permitía por su despliegue territorial, desde sus primeros años el INM nunca logró establecer mecanismos para recolectar, sistematizar y analizar la información generada a partir del quehacer institucional ${ }^{7}$ sobre rutas de tráfico de personas y redes de traficantes, entre otras razones, porque no estaba claramente establecido que, como parte de la Secretaría de Gobernación (Segob), el Inm tuviera facultades de investigación en esa materia. En efecto, en el ámbito de atribuciones de la Segob, las facultades para investigar estaban asignadas al Centro de Investigación y Seguridad Nacional (Cisen); sin embargo, el hecho mismo de que el Cisen contara, y cuente, con tales facultades no necesariamente implicaba que dentro de su agenda de seguridad nacional el tema migratorio haya sido un asunto relevante, como empezó a serlo a partir de $2001 .^{8}$

El problema con esa división tajante de atribuciones es que las dependencias que producían información no tenían facultades de investigación y las que investigaban no contaban con insumos suficientes para hacerlo; en

${ }^{5}$ El 31 de agosto de 1998 se creó la Coordinación Jurídica, de acuerdo con lo dispuesto en el artículo 53 del Reglamento Interior de la Secretaría de Gobernación.

${ }^{6}$ Generalmente una delegación regional comprendía dos estados de la República, por ejemplo, Tlaxcala y Puebla conformaban la Delegación Regional de Puebla.

7 Específicamente la que se deriva de los procesos de aseguramientos.

${ }^{8}$ A partir de los ataques a Estados Unidos el 11 de septiembre de 2001, tanto este país como su zona de influencia inmediata reevalúan el tema migratorio como parte fundamental de la seguridad nacional. En el caso de México, ello ocurre sobre el acuerdo previo, llamado Plan de Acción para la Cooperación sobre Seguridad Fronteriza, el cual fue anunciado por el subsecretario de Relaciones Exteriores de México, Enrique Berruga, el 28 de junio de 2001. 
tanto, la autoridad investigadora por excelencia en materia penal, de acuerdo con el sistema legal, era el MP, ${ }^{9}$ y éste no tenía entre sus funciones la de retroalimentar a otras dependencias con información relevante que ayudara a reformular estrategias institucionales. Lo que ocurría era una falta de conexión entre las dependencias federales.

Se entiende que ese enfoque de la administración pública sobre la división tajante de competencias partía del supuesto erróneo de que, al ser todas integrantes del Gobierno federal, tendrían esquemas de cooperación y coordinación para atender fines comunes, situación que en la práctica ocurría sólo en algunos ámbitos, ${ }^{10}$ pero no para la atención de los flujos migratorios de extranjeros indocumentados. En este sentido, lo que se tenía era un cúmulo de información desagregada y almacenada en cada dependencia, que por sí sola no explicaba nada y menos aún permitía generar un diagnóstico claro para la elaboración de políticas públicas integrales de atención al fenómeno en cada fase, según su complejidad y evolución.

Dado que no se tenía un sistema interno en el INM o interinstitucional que permitiera el intercambio permanente de información entre la dependencia que generaba la información y la encargada de hacer la investigación de inteligencia, sobre detenciones, tráfico y redes delictivas, poco podía esperarse sobre la elaboración de estrategias de largo plazo o aplicación con sentido prospectivo de medidas coyunturales para la atención de la migración indocumentada y el tráfico de personas.

Lo más relevante que se conocía sobre las rutas de tráfico en la administración pública federal eran los mapas que casi todas las dependencias de seguridad dibujaban a lo largo y ancho del país para tratar de explicar lo

9 El Ministerio Público no investiga más allá de un caso y cuando logra hacerlo, como es sabido, difícilmente progresan las querellas, pues, aunque sus facultades son amplias, sus capacidades son estrechas y su presupuesto insuficiente, para no hablar de prácticas que se apartan del proceder debido.

${ }^{10}$ A diferencia de la Coordinación de Control y Verificación Migratoria, la Coordinación de Regulación Migratoria, hasta 2000, tenía mecanismos claros y coordinados con otras dependencias para regular adecuadamente el flujo migratorio documentado. No así en materia de flujos indocumentados, donde el INM nunca logró articular mecanismos de atención interinstitucional. 
evidente: todas las rutas se originaban en el sur, se desagregaban en el centro y terminaban en las fronteras del norte de México (aunque no se veía que, de vuelta, esas mismas rutas servían para el traslado de armas, dinero y otros bienes, ilícitos o no). Sin embargo, eran materiales intuitivos, elaboraciones casi domésticas, carentes de la precisión georreferencial técnicamente posible.

No se conoce cuál pudo ser la metodología ${ }^{11}$ para el levantamiento de datos que llevara a algunas dependencias de seguridad a determinar la existencia de una ruta u otra. Hasta $2004^{12}$ no se hizo un trabajo sistemático para investigar redes de tráfico o establecer una cooperación interinstitucional entre la dependencia que administra y la que investiga para entrevistar a los migrantes extranjeros con el fin de conocer datos específicos sobre los costos, redes y contactos ${ }^{13}$ que pudieran ayudar a la definición de estrategias de atención claras, permitieran a la autoridad mantener su presencia y garantizaran la vida social en la legalidad.

Durante el primer año de la presidencia de Fox, el INM no contaba con información sobre rutas de tráfico de personas, ${ }^{14}$ y menos aún tenía cifras fidedignas sobre sus propios asegurados, ${ }^{15}$ como se dice en el argot guberna-

${ }^{11}$ El trazo de rutas generalmente obedecía a que, si un grupo de indocumentados centroamericanos era detectado en cualquier estado, se trazaba una nueva ruta desde ese estado hacia cualquier otro del sur, sin mayor detalle. Como cada dependencia hacía lo mismo, el Cisen trazaba sus propias rutas, la policía hacía lo mismo, pero el inM no se atrevía a hacer lo propio, simplemente daba por sentado que si las dependencias encargadas de seguridad lo decían, debía ser verdad.

${ }^{12}$ Antes de 2000 y hasta 2005.

${ }^{13}$ Con esto sólo se quiere señalar que no existió sistematización de información y de ninguna manera se niega que no hayan existido casos.

${ }^{14}$ Aunque existan cifras oficiales sobre el número de eventos de detención de migrantes indocumentados, éstas no pueden tomarse como totalmente confiables, toda vez que el método para contar a los detenidos en aquellos años, con frecuencia daba lugar a contar a un asegurado varias veces, según el número de estaciones donde estuviera la persona durante su detención. Cada delegación en cada informe contaba el número de detenidos que había atendido, lo que estaba bien. Empero, al hacerse el agregado nacional no se consideraba que un mismo asegurado podría haber sido contado para ese mismo mes en otra delegación donde hubiera estado previamente en resguardo gubernamental. Para ver la complejidad en la construcción del dato estadístico se recomienda consultar Casillas (2012).

${ }^{15}$ De hecho, se puede hablar de que existen tres momentos distintos en la generación de datos estadísticos sobre migrantes indocumentados en México en el inM: 1) la apreciación, con nulas posibilidades de cotejo; 2) la estimación, que se practica de manera retrospectiva, 
mental cuando se habla de migrantes extranjeros detenidos por carecer del permiso correspondiente, de tal suerte que con frecuencia se presentaba el caso de que cada dependencia de seguridad manejaba cifras elaboradas con criterios propios y sin que ninguna ellas citara sus fuentes; con el tiempo y la llegada de nuevas generaciones, el propio InM desconocería las cifras que previamente emitiera el mismo instituto.

Hasta 2004, pese a que el INM podía generar información de inteligencia a partir del desempeño de sus funciones, esta institución mantuvo una posición relegada en temas de investigación sobre tráfico de personas por ser una dependencia administrativa, sin funciones definidas en materia de investigación ${ }^{16}$ y fuera del ámbito del gabinete de seguridad nacional. ${ }^{17} \mathrm{Su}$ función era emitir resoluciones, prestar servicios, no obstante que desde 2002, en el Reglamento Interior de la Segob, se le otorgaban facultades al INM para investigar a extranjeros, aunque nunca para llevar a cabo investigaciones respecto a la delincuencia, puesto que eran funciones exclusivas del Ministerio Público Federal (MPF).

Por lo anterior, no se conocen documentos o análisis sobre estudios que refieran cuáles eran los esquemas de operación de las redes de traficantes de migrantes antes de 2000; las únicas constancias sobre la actuación de la autoridad migratoria hasta 2004 respecto a las medidas adoptadas sobre el tráfico de personas son las referencias internas — desagregadas en cada Delegación tanto del INM como de la PGR - sobre las querellas presentadas en cada Delegación, sin que dejen de ser atendidos como casos aislados y con las vicisitudes y limitaciones antes expuestas.

de 2008 hacia atrás, hasta 2002 aproximadamente, y cuenta con datos verificables pero carentes de amplia representación territorial o de continuidad en el tiempo, y 3) el dato duro verificable, con representación por entidad y con niveles de desagregación novedosos.

${ }^{16}$ Artículo 57 XVIII del Reglamento Interior del INM: «Investigar si los extranjeros cumplen con las obligaciones migratorias establecidas, y en caso de violación a las disposiciones sobre la materia, presentarlos ante las autoridades competentes».

17 En algunos casos, sólo aquéllos que se daban en flagrancia o por la gravedad del asunto, las resoluciones podrían desvelar alguna información que terminaría en una querella por parte del INM; sin embargo, éstos serían casos excepcionales porque investigar a los migrantes y sus propias circunstancias no ha sido una tarea institucional.

$54 \frac{\text { PRIMER SEMESTRE } 2015}{\text { MIGRACIÓN Y DESARROLLO NÚM. } 24}$ 
Hasta 2004, si bien no existía un trabajo sistemático sobre los flujos indocumentados y menos aún sobre los de origen centroamericano, ${ }^{18}$ había conocimiento de la existencia del traficante, cuya finalidad es la de prestar un servicio de traslado a cambio del pago de honorarios (a quien por lo regular se le confunde con el pollero, que en esencia es, o fue, un migrante que guía a un novicio del lugar de residencia hasta el de destino, de manera solidaria, así sea que reciba una compensación económica, pero no como actividad comercial o contractual ex profeso; esta confusión permitía que entre los empleados de Migración se generara cierta tolerancia hacia el traficante, al confundirlo con el pollero). Empero, dicho conocimiento no tenía su origen necesariamente en un trabajo sistemático de inteligencia, sino en el hecho real y concreto de la función de control y verificación de la autoridad migratoria en carreteras y puntos de revisión terrestres, es decir, a partir del encuentro con la realidad, y no como resultado de un trabajo estratégico para saber de, e incidir en, el tráfico de migrantes. ${ }^{19}$

Estas razones y prácticas constituyen las razones que llevaron, en parte, a la descomposición interna del INM (de mayor visibilidad social desde el asesinato de 72 migrantes en San Fernando, Tamaulipas, en agosto de 2010) y su incapacidad jurídica e institucional para responder oportuna y eficientemente ante la complejidad lograda en el tráfico internacional de migrantes y de la incursión del crimen organizado en dicha actividad. De ahí las interrogantes: ว̇qué clase de política migratoria podía formular el gobierno mexicano para atender correctamente el tráfico de migrantes de paso ante esas limitaciones, además de que es poco lo que sabía y este conocimiento estaba disperso y metodológicamente elaborado con propósitos y medios que no eran complementarios entre sí? , żcómo podría el gobierno mexicano anticiparse a la espiral de violencia y complejidad del fenómeno si no había una adecuada coordinación para que el responsable (INM), el coadyuvante

${ }^{18}$ Estos flujos, por su volumen y, sobre todo, porque nunca han sido considerados un riesgo para la seguridad nacional, no son de interés especial en el ámbito gubernamental, hasta el punto que durante muchos años no dejaron de ser un número en la estadística general.

${ }_{19}$ Ilícito en ambos sentidos, no sólo de quien traficaba, sino del propio migrante, pues hasta 2008 la Ley General de Población criminalizaba a los migrantes. 
(Cisen) y el investigador (MPF) hicieran un frente común para atender el problema? La explicación es la misma que se expuso al inicio: no era prioridad de las autoridades mexicanas elaborar una agenda sobre la migración indocumentada de paso. Así de sencillo y grave.

En sí mismo, el problema no corresponde del todo a la institución que tiene mayores facultades para coordinar los servicios migratorios, el INM. Se trata de un conflicto en la definición de atribuciones en la estructura de la administración pública, de suerte que de continuar el modelo prevaleciente hasta la fecha (2015), ninguna ley, reglamento o disposición de menor jerarquía podrá reunir y dirigir los esfuerzos gubernamentales en esta materia. En ese contexto, cualquier información que pudiera existir sobre este tema se encuentra dispersa y sin posibilidad de servir para entender las evoluciones de los flujos migratorios y de las redes de tráfico que dan lugar a articulaciones con redes delictivas de alta peligrosidad.

\section{Marco legal: de la persecución \\ A LA DESPENALIZACIÓN TARDÍA}

La Ley General de Población (LGP) publicada el 7 de enero de 1974, vigente desde entonces y hasta mayo de 2011, tuvo a lo largo de su existencia 14 reformas legales, de las cuales sólo dos incidieron de manera decisiva en el marco regulatorio de la migración indocumentada: las de 1999 y 2008.

Desde la publicación de la primera versión de 1974, se establecían sanciones para penalizar los flujos de migrantes indocumentados. En el artículo 103 de la LGP se señalaba lo siguiente: «Se impondrá pena de hasta dos años de prisión y multa de trescientos a cinco pesos al extranjero que se interne ilegalmente en el país».

En tanto que para casos de reincidencia, el artículo 98 preveía una sanción mayor al establecer:

Se impondrá pena de diez años de prisión y multa de hasta cinco mil pesos

al extranjero que habiendo sido expulsado se interne nuevamente a territorio

$56 \frac{\text { PRIMER SEMESTRE } 2015}{\text { MIGRACIÓN Y DESARROLLO NÚM. } 24}$ 
nacional sin haber obtenido acuerdo de readmisión. Igual sanción se aplicará al extranjero que no exprese u oculte su condición de expulsado para que se le autorice y obtenga un nuevo permiso de internación.

Las sanciones tuvieron pocas variaciones en las subsecuentes reformas y estas condiciones prevalecieron hasta la reforma de 21 de julio de 2008, mediante la cual se modifica, entre otros, el artículo 118 y 125 de la LGP con la finalidad de despenalizar la migración indocumentada de extranjeros. De acuerdo con este nuevo precepto legal (artículo 118), los migrantes indocumentados únicamente se harían acreedores a una multa de 20 a 100 salarios mínimos vigentes en el Distrito Federal, en tanto que el artículo 125 prevenía la aplicación de sanciones penales en caso de tipificación de otros delitos (para efectos prácticos, dicha sanción no se aplica; resulta más económico y expedito deportar al indocumentado detenido que iniciar y realizar todo el proceso jurídico administrativo para sancionar al infractor y lograr que éste pague la multa).

Es importante señalar que hasta 1999, la materia migratoria fue exclusivamente facultad y competencia de la Segob y de la Secretaría de Relaciones Exteriores (SRE), como autoridad coadyuvante en el exterior. Sin embargo, ese año se llevó a cabo una importante reforma ${ }^{20}$ con motivo de la creación de la entonces Policía Federal Preventiva (PFP), corporación a la que se le otorgaron atribuciones específicas en materia de verificación migratoria en todos los puntos de internación y dentro del territorio nacional. Para tal efecto, se modificaron los artículos 16, 17 y 151 de la LGP, que en conjunto establecen la prioridad del INM y la PFP para inspeccionar la entrada y salida de personas en cualquier forma que lo hicieran y en cualquier punto de internación. De manera exclusiva, el artículo 17 otorgaba a la PFP todas las facultades de inspección dentro de territorio nacional ${ }^{21}$ de toda persona en tránsito internacional, medida que en sentido estricto enfatizó el sentido

${ }^{20}$ Diario Oficial de la Federación, 4 de enero de 1999.

${ }^{21}$ La diferencia de competencias no es de menor relevancia, pues mientras el inM estaba sujeto a un procedimiento interno - no se podía establecer un punto de revisión interno sin previa comisión de autoridad competente- para realizar tareas de verificación en puntos 
persecutorio a la migración indocumentada, al otorgar mayores facultades a la PFP para revisar y detener a cualquier migrante sin que mediara orden de verificación previa.

La reforma, mediante la cual se facultó a la policía para entrar de lleno al ejercicio del control migratorio, estuvo acompañada de una medida paralela: la cancelación de permisos de portación de armas para los agentes migratorios, dejando de esta manera el uso de la fuerza exclusivamente a la PFP y las funciones administrativas al INM. Ante la incapacidad legal para portar armas, los operativos sorpresa (llamados volantas) en cualquier lugar de las rutas migratorias ya no fueron posibles de hacer con seguridad para el personal operativo, pues ante un transporte que fuera encabezado por gente armada, simplemente ese personal indefenso tenía, como lo hizo con frecuencia, que franquear el paso y luego dar parte a la autoridad judicial, sin que exista registro de que esos reportes hayan conducido a detenciones de traficantes. En Tamaulipas este proceder fue frecuente, pero no sólo en esa entidad.

Todo lo relativo a los asuntos de indocumentados, en consecuencia, desde hace cuando menos 15 años, estuvo sujeto a dos estrategias no siempre homogéneas y muchas veces opuestas: por un lado, la promovida desde el enfoque policial con su propia autonomía y visión de la migración y, por otro, el enfoque administrativo que promovió el INM, con resultados poco efectivos. Sobre esta dualidad ha discurrido la suerte de la migración indocumentada y el destino de los propios migrantes.

En 2008 el gobierno mexicano decidió promover una reforma a la LGP en materia de migración indocumentada para despenalizar con sanciones de privación de la libertad a los extranjeros que ingresaran a México sin papeles. La decisión resultó un éxito mediático en cuanto que dio congruencia al discurso político, pero no como una disposición que impactara en las condiciones generales de los migrantes, habida cuenta de que hacía más de 10 años que esa determinación había dejado de ser viable y, por ende, era de nula

distintos a los puntos de internación (carreteras) la Policía Federal sí tenía autorización para detener migrantes sin sujetarse a ninguna formalidad. 
aplicación. Por lo anterior, se puede decir que la reforma de facto se hizo con anticipación a la norma de jure. No obstante, vale reconocer el esfuerzo de ir logrando correspondencia entre realidad y norma jurídica.

La reforma de 2008, si bien despenalizó la migración para quien ingresaba de manera indocumentada, no lo hizo respecto a quienes lo hacían utilizando documentación falsa. A favor de la visión administrativa instrumentada por el INM, se puede afirmar que esta sanción también tiende a desaparecer de facto, sobre todo para los migrantes centroamericanos. La razón de fondo para que la administración haya sido omisa respecto a las sanciones dispuestas en la LGP hasta el 25 de mayo de 2011 obedecía a las mismas que motivaron la despenalización: aplicación inviable. ${ }^{22}$ En este sentido, debe hacerse notar que desde 2008 las diferencias abismales entre los preceptos legales y las disposiciones administrativas operaron a favor de los migrantes, pero sobre todo quienes más se aprovecharon de esas diferencias han sido, y son, las redes delictivas.

Si bien la reforma no dejó de ser importante, al dar mayor certeza jurídica a los migrantes, estuvo muy lejos de responder a las necesidades del INM $y$ de los propios migrantes. No se comprenden las razones que motivaron al Ejecutivo a mantener la vigencia de sanciones pecuniarias que de facto habían dejado de aplicarse de manera generalizada a los migrantes centroamericanos, por inviables, al incidir directamente en el entorpecimiento de la repatriación de éstos.

Ciertamente, la reforma satisfizo un aspecto político, pero no logró normar el fenómeno migratorio ni otorgar mayores beneficios a los migrantes, así como tampoco dio al INM mayor margen de maniobra en atención al volumen creciente de migrantes centroamericanos. La incapacidad de éstos para cubrir cualquier multa por ínfima que fuera, ${ }^{23}$ la falta de capacidad

22 Es frecuente que entre los migrantes centroamericanos el traficante distribuya credenciales mexicanas para votar ya sea falsas o robadas con la finalidad de sorprender a la autoridad en caso de revisión y solamente cuando éstos son detenidos por la policía y puestos directamente ante el MPF. En caso de detención por parte del INM, se privilegian criterios administrativos por encima de los judiciales y son inmediatamente repatriados sin que sean acreedores a sanción alguna.

${ }^{23}$ Pese a que se reafirmó la sanción pecuniaria, para el caso particular de los migrantes indocumentados centroamericanos, es letra muerta. 
instalada en las estaciones migratorias para albergar por largos periodos a los migrantes insolventes, y la inviabilidad para presentar ante el MPF al creciente flujo de migrantes indocumentados, multar o presentar ante el MPF a los reincidentes y portadores de documentación falsa, hicieron que las disposiciones de los artículos 118 y 125 reformados en 2008 (algo que se mantiene en la Ley de Migración de 2011) hayan nacido muertas para el caso específico de los migrantes centroamericanos indocumentados detenidos.

En los hechos, para el caso de los migrantes centroamericanos, estas diligencias ministeriales terminaron en simulación de la autoridad, que los presentaba por procedimiento para eximirse de responsabilidad, y del MPF, que evitaba a toda costa encontrar elementos para consignar las averiguaciones previas, ante la amenaza de tener bajo su custodia a los migrantes. ${ }^{24}$

Sobre lo anterior, es pertinente destacar que la inobservancia de las sanciones pecuniarias previstas (artículo 118) a los migrantes indocumentados centroamericanos, paradójicamente, terminó siendo un beneficio más real y tangible que la despenalización de la reforma de 2008.

En este sentido, debe destacarse que, más allá de la despenalización de la migración y dada la vulnerabilidad de las circunstancias de los migrantes, lo que realmente opera a su favor es: a) agilizar la resolución de las expulsiones; b) facilitar las repatriaciones; c) despresurizar las estaciones migratorias; ${ }^{25}$ d) evitar el riesgo de motines, ${ }^{26} \mathrm{y}$ e) incrementar la seguridad en las estaciones migratorias.

Por lo anterior, mientras el conjunto de dependencias federales vivía procesos de ajuste que no abonaban a una mejor coordinación interinstitucional, las modificaciones legales, aunque contradictorias, flexibilizaban (cuando no alejaban) el control estatal sobre los flujos indocumentados, que

${ }^{24}$ Migrantes que ninguna dependencia quiere tener, pues deben proveérseles alimentos cuando menos durante el término de 48 horas si no se trata de un migrante solvente, cosa que no ocurre con los centroamericanos.

${ }^{25}$ En el caso particular de la estación migratoria El Vergel, acondicionada para poco más de 400 personas, entre 2000 y 2004 registraba poblaciones de entre 800 y hasta 1,500 detenidos.

${ }^{26} \mathrm{La}$ alta concentración de migrantes y la larga permanencia de éstos por asuntos procedimentales como los referidos, motivó que entre 2000 y 2006 se incrementaran los motines en el interior de las estaciones migratorias, hechos poco conocidos públicamente.

$60 \frac{\text { PRIMER SEMESTRE } 2015}{\text { MIGRACIÓN Y DESARROLLO NÚM. } 24}$ 
seguían engrosando y quedando más cercanos y desprotegidos frente a la mano delictiva (Strange, 2003).

\section{Migración internacional: ¿UN TEMA DE SEGURIDAD NACIONAL?}

A partir de 2001, después de los ataques en territorio estadounidense, la Segob inició un proceso de vinculación entre dos entidades de la misma dependencia que previamente no habían tenido una relación de trabajo directa: ${ }^{27}$ el InM y el Cisen. De esta manera, se estableció que el INM colaborara exclusivamente ${ }^{28}$ con información para la elaboración de una agenda de riesgos sobre el tema migratorio. Sin embargo, siguiendo el esquema de fragmentación de facultades en la administración pública en estas materias, no se estableció que el InM también pudiera participar en el diseño de la agenda de riesgos para lograr mejores resultados en la ejecución de la misma. Ello tendría repercusiones en la actuación diaria.

Desde 2001, la agenda de riesgos ha estado orientada a vigilar los flujos migratorios documentados por nacionalidades, es decir, recayó sobre las funciones reguladoras de la institución, y no necesariamente sobre las implicaciones y riesgos que pudiera representar la migración indocumentada, no obstante que el gobierno de México carece de un efectivo control fronterizo en el sur del país. Para atender esta problemática no resuelta, el inm impulsó diversos proyectos, entre los cuales estaba el de Frontera Sur ${ }^{29}$ que, por sus

${ }^{27}$ El Cisen, como dependencia investigadora, no establecía una relación de trabajo horizontal y compartido con otras dependencias. Esto no se refiere a las relaciones cupulares que pudieran existir entre los titulares en la materia, sino a la instrumentación de medidas interinstitucionales que conlleven trabajo cotidiano y una agenda compartida.

${ }^{28}$ El sentido de la colaboración es reafirmado en el acuerdo mediante el cual se reconoce al INM como entidad de seguridad nacional.

${ }^{29}$ Desde la perspectiva de seguridad no tenía ninguna viabilidad un proyecto que por principio empezaba por trasladar las fronteras y los servicios migratorios al interior del país en lugar de ubicarlos en las fronteras jurídicamente establecidas del Estado-nación; por eso no contó con la aceptación de las instancias de seguridad e inteligencia nacionales y quedó como 
limitaciones conceptuales en materia de seguridad, no pasó de ser una propuesta con limitadas implicaciones concretas en el presupuesto y labor institucional. No obstante, desde 2008 las corrientes migratorias centroamericanas se han engrosado con migraciones extracontinentales de asiáticos y africanos; no es que éstas no ocurrieran previamente, sino que eran de menor volumen y ocurrían de manera paralela a las centroamericanas, pero se subvaloraba la importancia estratégica de las redes de traficantes que los introducían y conducían por suelo mexicano (Celorio, 2014). Su peligrosidad quedaría evidenciada de manera irrefutable con el asesinato de los 72 migrantes de San Fernando en 2010, en donde había un nacional de India, y ratificada con las otras fosas descubiertas también en San Fernando en 2011. Es decir, en esos 72 migrantes asesinados estaba la evidencia irrefutable de la labor convenida entre redes de traficantes centroamericanas y extracontinentales.

En 2000 el INM, después de haber consolidado su despliegue territorial a través de sus delegaciones, instrumentó diversos acuerdos delegatorios de facultades, con la finalidad de desregular y desconcentrar la prestación de servicios a través de las delegaciones regionales. Sin embargo, tras lo ocurrido el 11 de septiembre de 2001 en Estados Unidos, y como parte de las medidas de emergencia ante riesgos desconocidos, se dio marcha atrás a la desconcentración de facultades (que permitía a los delegados resolver cualquier trámite migratorio sin excepción de nacionalidad) para centralizar nuevamente las decisiones sobre internación de nacionalidades del denominado grupo uno o de permiso previo, comúnmente conocido como nacionalidades restringidas, ${ }^{30}$ sobre la base de la recién elaborada agenda de riesgos a la seguridad nacional de México en materia migratoria, la cual no era otra cosa que la extensión de la agenda de riesgos del gobierno estadounidense en la misma materia en suelo e instituciones gubernamentales mexicanos.

mera propuesta del INM, por cierto no compartida internamente por la totalidad de las coordinaciones del propio instituto ni de quienes estaban al frente de las delegaciones estatales.

${ }^{30}$ Para mayor referencia véase su publicación en el DOF.

$62 \frac{\text { PRIMER SEMESTRE } 2015}{\text { MIGRACIÓN Y DESARROLLO NÚM. } 24}$ 
Es frecuente que la administración pública mexicana en ciertas materias tome como parámetros de referencia algunas prácticas del gobierno estadounidense para aplicarlas como propias en el país. ${ }^{31}$ En lo que concierne a la agenda de riesgo instrumentada desde 2001, se actuó de igual manera. Desde esta perspectiva, se asumía que los agentes de riesgo para la seguridad nacional de Estados Unidos eran los mismos que los de México, tanto en la identificación de los objetivos de riesgo como en los mecanismos para evitarlos. ${ }^{32}$

De lo anterior se puede inferir que la decisión de convertir al INM en entidad de seguridad nacional el 18 de mayo de 2005, casi cuatro años después de lo ocurrido en Estados Unidos, no deviene de una necesidad propia o de un riesgo inminente a la seguridad interna, sino como resultado de la transformación de la seguridad regional de América del Norte para vigilar los flujos migratorios de su interés; ${ }^{33}$ en otras palabras, como parte de los acuerdos bilaterales de cooperación para realizar una serie de tareas encaminadas a evitar el posible tránsito de agentes de riesgo cuyo destino final fuera el territorio estadounidense, con excepción de los migrantes centroamericanos que nunca han sido considerados agentes de riesgo, aunque se intente disminuir el flujo indocumentado, pero por otras razones.

Desde 2001 a la fecha no se conoce públicamente de algún riesgo inminente a la seguridad nacional como para urgir la transformación del INM en entidad de seguridad nacional. En ese sentido, los argumentos ${ }^{34}$ del acuerdo

${ }^{31}$ El ejemplo más claro es el de la expedición de la visa mexicana. Durante muchos años la lista de requisitos no dejó de ser un símil de la estadounidense. El 1 $1^{\circ}$ de mayo de 2010 entró en vigor la medida que permite a los extranjeros con visa estadounidense ingresar a México con la misma certeza jurídica que quienes tramitan una visa mexicana.

32 Durante años se creyó que los agentes de riesgos tenían que vigilarse desde la trinchera más fácil de registrar e identificar, la del flujo documentado. Se partía del supuesto de que el terrorista solicitaría una visa para viajar a México y lo haría con su propia identidad, sin tomar en consideración que también era posible que ingresara caminando o por cualquier otro medio con documentos falsos. El ejemplo más claro de esto fue el ingreso al territorio nacional del terrorista cubano-venezolano Posada Carriles.

${ }^{33}$ Cualquier nacionalidad de origen árabe.

34 «La seguridad nacional tiene como metas principales velar por la protección y preservación del interés colectivo, evitando en lo posible o minimizando cualquier riesgo o amenaza a la integridad física de la población y de las instituciones...", y propone "concebir la seguridad nacional desde una visión amplia destinada a prever y hacer frente a situaciones que ame- 
por el cual se reconoce al INM como tal permiten afirmar que responden a un modelo de operación y a necesidades fuera del territorio nacional.

En 2001, Estados Unidos toma una serie de medidas que modificarán la agenda en las relaciones bilaterales con México y que impactarán profundamente en ciertas áreas de la administración pública federal. Redimensiona los riesgos de la migración documentada e indocumentada en los términos ya mencionados previamente, en tanto que a nivel externo establece nuevos esquemas de cooperación estratégica, ${ }^{35}$ especialmente con México, en diversas materias para reforzar su seguridad. Dentro de la gama de cuestiones están de manera preponderante los asuntos migratorios. ${ }^{36}$

Esta circunstancia modifica para el INM las condiciones de operación interna y relaciones externas. Por primera vez se crean mecanismos para tomar el control de procesos de recolección, automatización ${ }^{37}$ y sistematización de

nacen la paz, la vigencia del orden jurídico, el bienestar y la integridad física de la población y que pongan en riesgo la permanencia de las instituciones o vulneren la integridad del territorio». "Que las atribuciones del Instituto Nacional de Migración tienen relación directa con la Seguridad Nacional, ya que de acuerdo con la Ley General de Población y el Reglamento Interior de la Secretaría de Gobernación, dicho Instituto tiene la facultad y responsabilidad de proteger y tutelar la seguridad nacional a través de la restricción de la emigración de nacionales (...) (;) prohibir la admisión de extranjeros cuando así lo determine el interés nacional; expulsar a los extranjeros en casos de que se atente en contra de la soberanía o la seguridad nacional; (...); investigar si los extranjeros cumplen con las obligaciones migratorias establecidas (...). Que para una adecuada realización de sus actividades y, con el fin de que éstas se encaminen dentro del contexto de la seguridad nacional, es necesario que exista una colaboración con el Centro de Investigación y Seguridad Nacional para que éste se constituya como la institución rectora de la Red Nacional de Investigación y por lo tanto funja como órgano de coordinación, definición y articulación de las acciones tendientes a la constitución y operación de la Red Nacional de Investigación (...)».

${ }^{35}$ El redimensionamiento de los riesgos de la migración, como cambios en la navegación aérea y las condiciones del viajero, de tal suerte que dentro de la estrategia global de Estados Unidos esté la de extender sus servicios de inteligencia a los aeropuertos de origen, de esta manera, a través de la agencia ICE, sus agentes revisan las listas de pasajeros con destino a Estados Unidos, desde Madrid, México o Beijing.

${ }^{36}$ Las fuentes refieren que hasta 2004 prevalecía una regla no escrita, en el sentido de que ninguna agencia extranjera podía establecer relación directa con ninguna dependencia del Gobierno federal que no fuera el centro de inteligencia de la Segob o con intermediación de ésta.

${ }^{37}$ Se impulsan proyectos tecnológicos como el siom para hacer más efectivo el control de los flujos documentados, aparentemente con apoyos del gobierno de Estados Unidos, que bien puede ser el antecedente del Plan Mérida. 
la información que genera, no con el propósito final de llevar a cabo trabajo de inteligencia o suplantar las funciones del Cisen, sino para tomar el control del eslabón de seguridad que le correspondía y que, por ser facultad exclusiva del INM, no pudo ser transferido, salvo alguna excepción, ${ }^{38}$ a ninguna otra dependencia por mucho que se desconfiara de la fortaleza institucional y se dudara de la honestidad de su personal. Sin embargo, debe señalarse que estos procesos no han logrado consolidarse ni por el lado de la tecnología ni por cuanto se refiere a la sistematización de la información que generan las delegaciones regionales ni las oficinas centrales. Quizá la pregunta de fondo no respondida en ese entonces, ni ahora, es żqué quiere decir seguridad nacional en la lógica propia del INM?

A nivel externo se establecieron mecanismos de cooperación directa, nacionales e internacionales, para la operación cotidiana de los flujos migratorios, de suerte que el hecho de que el INM se haya convertido en una entidad de seguridad nacional para formar parte de la red de investigación coordinada por el Cisen, no ha tenido ninguna repercusión a nivel interno, es decir, a nivel de la elaboración de políticas públicas. Por el contrario, el inM en la presidencia de Felipe Calderón cayó en la orfandad — visto desde el contexto del gabinete de seguridad- $y$, desde esa posición, se convirtió en una fuente de información externa que abonaba más a la agenda de riesgos del gobierno estadounidense ${ }^{39}$ que a los intereses nacionales.

${ }^{38}$ Una herramienta fundamental para verificar las listas de pasajeros son las denominadas listas APIS, que son la versión electrónica de las listas que la LGP exigía a las aerolíneas. El uso de éstas no fueron asignadas al INM por un asunto de desconfianza institucional y fueron entregadas a Aduanas, mediante una reforma que le da atribuciones para administrar listas de pasajeros, mandato que previamente la LGP había otorgado al INM. De acuerdo con la división interna de facultades, las aduanas en México únicamente revisan mercancía. Pero como se trata de un proyecto bilateral con Estados Unidos, se hizo una adecuación a modo que desde la perspectiva estadounidense su homólogo en México sea Aduanas y no el inM; otra pequeña concesión del gobierno de México a su vecino del norte.

${ }^{39}$ La comisionada del INM en ese entonces, Cecilia Romero, hizo declaraciones del siguiente tenor: «sí se permitió que hubiera alguna entrevista a algún indocumentado, pero no centroamericanos, más bien de otras nacionalidades», y aclaró que las autoridades estadounidenses no entraban a entrevistar a todos los que quisieran, ya que se tenía que seguir un protocolo muy concreto, el cual tenía que ser validado por las autoridades mexicanas. "Entonces sí se permitía que se hiciera una breve entrevista, con un cuestionario realizado por noso- 


\section{SOBRE LA DESCOMPOSICIÓN INSTITUCIONAL DEL INM}

El INM, desde su creación en 1993, define claramente dos áreas estratégicas para la atención de la migración: la Coordinación de Regulación Migratoria, para atender la migración documentada, normar los criterios de internación y autorizar la internación de los extranjeros, entre otras funciones; para controlar y verificar los flujos migratorios documentados e indocumentados, se creó la Coordinación de Control y Asuntos Jurídicos. ${ }^{40}$ La evolución de las medidas para favorecer corrientes migratorias documentadas, ya sea con fines turísticos y por motivos de residencia temporal o definitiva, ha sido notable a partir de las medidas de desregulación instrumentadas progresivamente desde el inicio del siglo XXI, si bien es cierto que su incidencia en el número total de extranjeros sigue siendo menor a 1 por ciento.

La falta de interés y la inoperancia institucional para el control de la migración indocumentada han contribuido, sin duda, en la configuración del problema del tráfico, que ha devenido en una espiral de violencia para los indocumentados; pero también han puesto de manifiesto cómo la incapacidad institucional ha favorecido y fortalecido a la delincuencia para hacerse del control de los migrantes. No menos relevante es el hecho de que, frente al debilitamiento institucional, distintos agentes migratorios terminen siendo colaboradores de la delincuencia organizada. ${ }^{41}$

La migración indocumentada ha sido parte de la realidad histórica mexicana. Un centroamericano que migra a Estados Unidos tiene que pasar por territorio mexicano de manera documentada o no. Así ha sido, lo han hecho por sus propios medios o con ayuda de un tercero; forma parte de una realidad durante el siglo xxy lo que va del Xxi. ${ }^{42}$ Frente a estas corrientes migratorias

tros, pero en un espacio distinto a las estaciones migratorias». Véase http://www.animalpolitico.com/2011/01/si-el-fbi-entrevisto-a-migrantes-en-mexico-pero-no-como-lo-revelowikileaks-cecilia-romero/

${ }^{40}$ Esta coordinación, en 1998 dio origen a la Coordinación Jurídica.

${ }^{41}$ El 9 de mayo de 2011, la SIEDo arraigó a seis agentes federales de migración, tras haber sido reconocidos por las víctimas de haberlos entregado a los Zetas.

${ }^{42} \mathrm{La}$ porosidad institucional de la frontera sur de México, con el tiempo, también ha favorecido las rutas de migración extracontinental, las fronteras de Chiapas y Tabasco ven pasar migrantes de todo origen.

$66 \frac{\text { PRIMER SEMESTRE } 2015}{\text { MIGRACIÓN Y DESARROLLO NÚM. } 24}$ 
había una presencia del Estado para contenerla o administrarla, ${ }^{43}$ como parte de las medidas vigentes hasta $2000 .{ }^{44}$

Dentro de todas las variables que convergen en este fenómeno histórico, migrantes, autoridad y traficantes, únicamente subsiste una constante con sus mismas características: los migrantes son los mismos de siempre y su volumen aumenta por factores diversos — crisis económicas, desastres naturales, etcétera- que agravan las condiciones económicas de las naciones y estratos medios y bajos, de manera que eso lleva a pensar que en la nueva configuración de fenómeno migratorio - marcado por la violencia y la inseguridad - lo que ha cambiado y empeorado el problema en los últimos años son los errores e involuciones de la política migratoria gubernamental y el perfeccionado modus operandi de los traficantes. En este nuevo escenario, también emerge otro actor muy destacado: la llamada sociedad civil, ${ }^{45}$ la cual requiere un estudio aparte (Casillas, 2011).

¿Qué había en materia migratoria hasta 2000? Una legislación que penalizaba la migración indocumentada, es decir, que criminalizaba al migrante y lo hacía perseguible; una presencia del Estado (el INM, PF y todas las policías) con autoridad suficiente para actuar como tal, para bien y para mal, pero con mayor capacidad para controlar ${ }^{46}$ los flujos migratorios. El engranaje operativo del sistema institucional en esos años permitía que ninguna ruta de tráfico o traficante pasara de manera independiente o por «la libre», es decir, sin participación de agentes del gobierno, todos debían pagar su cuota a la autoridad. ${ }^{47}$ Los traficantes tenían la imperiosa necesidad de entenderse con

${ }^{43}$ Entiéndase con esto la facultad discrecional de la autoridad migratoria para asegurar al extranjero o bien permitir su paso mediante la aceptación de sobornos.

${ }^{44}$ No había una segunda lectura al mandato de la LGP que asegurar al indocumentado y proceder a su expulsión como lo establece ésta. En términos administrativos se consideraban las cifras de aseguramientos como sinónimo de eficiencia administrativa.

${ }^{45}$ Las instituciones pierden credibilidad y respeto cuando se empieza a negociar la ley, y en el caso de la LGP, ésta se empezó a negociar con la Iglesia católica a partir de 2000, cuando se convino no llevar a cabo visitas de verificación a los albergues y casas del migrante, que en México son, en poco más de 90 por ciento, de impronta católica.

${ }^{46}$ En este caso, controlar significa que lo hacía con fines institucionales y de tráfico, es decir, controlaban ambas partes.

${ }^{47} \mathrm{La}$ autoridad no era sino los agentes migratorios, especialmente de Chiapas; usaban la investidura y las facultades para sus negocios personales porque con salarios mensuales de 4,000 pesos de entonces, resultaba inexplicable su enriquecimiento. $\frac{2015 \text { PRIMER SEMESTRE }}{\text { MIGRACIÓN Y DESARROLLO VOL. } 13} \mathbf{6 7}$ 
ellos, pero también con otros que en su calidad de autoridad hacían funciones de control migratorio extralimitándose en sus atribuciones, como las policías municipales y estatales. ${ }^{48}$

A partir de 2005 el INM toma medidas ${ }^{49}$ para hacer valer las disposiciones legales sobre las facultades exclusivas de las autoridades federales (INM, PF) en materia de detenciones, excluyendo con ello a otras dependencias gubernamentales que, de manera irregular y permanente, venían haciendo un trabajo de «autoridad». A partir de este momento y de otras situaciones que convergen, se configura un nuevo escenario que en el mediano plazo abonaría a la descomposición que aqueja al propio INM y su actuación en la atención a la migración indocumentada:

1) Se debilita la presencia institucional del Estado en las zonas fronterizas, es decir, la suma de fuerzas institucionales que conformaban el INM, PF, policías estatales y policías municipales — que lo mismo servían para asegurar que para traficar- se rompe por completo. ${ }^{50}$

2) La desautorización a los policías municipales y estatales para llevar a cabo funciones migratorias que habían efectuado por años implicó un alejamiento de autoridad, pero no un retiro del personal operativo de la actividad. ${ }^{51}$ Del nicho migratorio se eliminó la presencia de la institución de seguridad, pero se «independizó» a los agentes físicos que la encarnaban. Así, al no poder ocuparse del asunto como autoridad — para detener o extorsionar- los agentes empezaron a incursionar en la extorsión al migrante a título personal

48 Para bien o para mal, todas las policías más el inM conformaban un bloque con poder frente a la migración y la delincuencia.

${ }^{49}$ Hacer la recepción de extranjeros con la correspondiente notificación al policía local de que se haría una queja al órgano interno correspondiente por llevar a cabo funciones para las cuales no era competente. En algunos casos, se les negó a las policías locales la recepción de los migrantes.

${ }^{50}$ Paradójicamente, años después, con motivo de la promulgación de la nueva Ley Migratoria, el presidente Calderón señaló: «La división de competencias no será, no debe ser ya un pretexto para no atender los delitos ofensivos, graves, contra quienes migran, como son el secuestro, la extorsión, el tráfico de personas, la violación y muchas otras».

${ }^{51}$ Como ejemplo está la policía municipal de Tierra Blanca, señalada por las víctimas de secuestro de internos y por entregarlos al jefe de la plaza de los Zetas. El 15 de marzo de 2010, fueron detenidos 90 policías municipales por vínculos con la delincuencia organizada.

$68 \frac{\text { PRIMER SEMESTRE } 2015}{\text { MIGRACIÓN Y DESARROLLO NÚM. } 24}$ 
o como delincuente coludido con las organizaciones delictivas, hasta devenir en empleado de las mismas.

3) Al mismo tiempo, ocurre que cuando las policías locales son replegadas como autoridad, ni la pF ni el INM tienen fuerza para contener el creciente problema, lo que facilita que los agentes físicos puedan ponerse al servicio de la delincuencia. A manera de ejemplo, para 2012, el INM tenía como personal de campo en el estado de Chiapas a 120 personas, 40 en volantas en Oaxaca y 15 en San Luis Potosí, por turno, cifras irrisorias si se tiene presente la densidad territorial de esas entidades, sus carreteras, red ferroviaria y configuración del suelo.

4) El detonante más grave de la medida tomada en 2005 fue que en el cambio de mandos en 2006, la nueva administración del INM tomó la decisión de «no actuar», es decir, dejó de hacer operativos para la detención de migrantes extranjeros indocumentados y se limitó a labores circunscritas al ámbito inmediato de las delegaciones regionales. Como consecuencia de ello, hubo una significativa reducción en eventos de detención, como lo muestran las estadísticas oficiales. ${ }^{52}$

El resultado de la cadena de errores y omisiones es el escenario que se observa y padecen los migrantes a partir de 2008 (aunque las autoridades actuales han logrado, con relativo éxito tender un manto de invisibilidad sobre él): una autoridad que, al dejar de cumplir sus obligaciones (Wacquant, 2010), abre los espacios para que entren de lleno otros actores sociales y la delincuencia organizada se haga del control de las rutas, de los flujos migratorios y, en consecuencia, del destino y la vida de los migrantes. El nicho social migratorio abandonado por la autoridad fue ocupado por las redes delictivas.

Hasta 2004 lo que había era un esquema ampliado de corrupción institucional y con eso tenía que navegar el traficante, pero no necesariamente en todos los casos el migrante. El funcionario de migración corrupto que controlaba el tráfico era complacido con dinero; ${ }^{53}$ el migrante sólo salía afec-

\footnotetext{
52 Se recomienda ver Casillas (2012).

${ }^{53}$ El móvil era la extorsión, no el secuestro o la privación de la vida.
} 
tado con la detención en caso de que su traficante no lograra buenas negociaciones con $\mathrm{la}(\mathrm{s})$ autoridad(es), de suerte que bajo este esquema, el traficante garantizaba por el mismo precio hasta tres intentos para pasar por territorio mexicano. En este sentido, la suerte del migrante estaba determinada por los negocios del binomio funcionarios públicos corruptos-traficantes tradicionales, cuyo peor escenario era la detención y la expulsión, pero no el riesgo de perder la vida, ${ }^{54}$ como sucedería al poco tiempo.

Hasta hace pocos años, el migrante huía de «la migra», mientras que con el cambio de estrategia gubernamental quedó sin escapatoria: fue entregado directamente a la delincuencia organizada y la posibilidad de que fuera detenido por migración se redujo de manera significativa. El INM, en virtud de sus políticas de «no hacer» en los espacios ilegalmente convenidos con actores religiosos, perdió el control de sus funciones. ${ }^{55}$

¿Qué medidas instrumentó el INM a partir de 2007 para administrar la migración indocumentada? Se configuraron distintos factores que facilitaron el rápido incremento de la violencia de los grupos delictivos, que pasaron de secuestros exprés de migrantes a secuestros masivos en trenes y al asesinato masivo de los mismos; fueron los primeros signos alarmantes de la crisis institucional del INM, que evidenció el informe sobre secuestros de migrantes de la Comisión Nacional de los Derechos Humanos (CNDH, 2009a).

Los elementos en la actuación del INM que se conjugaron de manera negativa fueron, básicamente, tres:

1. Política de no hacer. Lo primero que se observa es una caída notable en el número de detenciones sobre las cifras sostenidas en los años anteriores. ${ }^{56}$

${ }^{54}$ Con esto no se quiere decir que lo que ocurría era mejor en términos institucionales, pues la corrupción en el INM ha sido un viejo lastre que no se ha podido remontar.

${ }^{55}$ En el análisis de lo que ha ocurrido con la transmigración y secuestros de migrantes, se cayó en una defensa insostenible al decir que no era competencia del INM el tema de seguridad pública, lo cual es una verdad a medias, pues por distintas razones el inM dejó de hacer labores propias de su mandato legal, acotó participaciones institucionales que le servían de contrapeso y privó de participación de otras que ya estaban involucradas en el proceso, liberando a actores físicos compenetrados en el modus operandi del tráfico de la migración. De ahí que sin tomar en cuenta estos equívocos internos resulte imposible elaborar una estrategia operativa de corto y mediano plazo en lo que es materia propia del INM.

${ }^{56}$ Se recomienda ver el artículo "La construcción del dato oficial...» ya referido.

$70 \frac{\text { PRIMER SEMESTRE } 2015}{\text { MIGRACIÓN Y DESARROLLO NÚM. } 24}$ 
Para 2007 el total de detenciones se redujo a 51,700. En la composición de las estadísticas destaca el hecho de que por primera vez el bloque de los cuatro países que históricamente han aparecido en los primeros cuatro lugares $(\mathrm{CA} 4)^{57}$ se vería acompañado de nacionalidades distintas, pues hubo un incremento en el número de cubanos $(1,438)$, ecuatorianos $(1,364)$ y brasileños $(1,067)$.

Hay pocas razones para pensar que de un año a otro cambiaron radicalmente las condiciones que favorecían la migración centroamericana (Zúñiga, 2010). Por el contrario, la creciente presencia de nacionalidades de América del Sur sugiere que la tendencia de la migración indocumentada se mantenía constante. Lo que se observa es un cambio en la estrategia institucional sobre qué hacer con sus funciones en materia de control y verificación migratoria; si el INM hiciera públicos sus archivos de las volantas, que los tiene en Excel, por entidad, por ejemplo, se vería cómo los cambios de ubicación, el número y frecuencia de ellas incide en el total de indocumentados asegurados.

A partir de la administración federal que iniciara labores en 2006 se instrumenta la medida de reducir la atención al flujo de migrantes de paso indocumentados. No hubo una instrucción precisa y escrita que lo determinara; sin embargo, la fuentes orales consultadas señalan que a diferencia de la administración que le antecedió, basada en la exigencia de resultados en detenciones, ésta privilegió una visión asistencialista — muy cercana a las posiciones de la Iglesia católica - de consentir que el migrante de paso transitara sin ser molestado. Para ello se llevaron a cabo medidas como la cancelación de puntos de revisión migratoria ${ }^{58}$ que durante años cumplieron una función de control. Esta decisión, con los años demostraría ser errónea y carente de sentido de responsabilidad administrativa.

${ }^{57}$ Nombre técnico que recibe el grupo de los cuatro países centroamericanos con mayor número de migrantes.

${ }^{58}$ Sólo por poner un ejemplo, el Manguito y el Hueyate, en Chiapas, que siempre fueron los primeros filtros de revisión, fueron cancelados. Ciertamente, en ellos había abusos y extorsiones. Al cancelar esos filtros migratorios se retiró la autoridad, pero quedaron las prácticas de abuso y extorsión, ahora por agentes liberados de la presencia y tutela institucionales. 
Antes del gobierno de Calderón no había ocurrido que un estado fronterizo, como Chiapas, que por razones geográficas siempre ocupó el primer lugar en detenciones por ser la gran puerta de ingreso a México, en 2007 tuviera 1,131 migrantes detenidos, ${ }^{59}$ es decir, sólo 2.2 por ciento del total nacional, por debajo de Tabasco (31.7 por ciento), Veracruz (17.9 por ciento), Oaxaca (8.7 por ciento), Tamaulipas (8.5 por ciento), San Luis Potosí (6.3 por ciento), sólo por mencionar esa ruta de migrantes centroamericanos de paso. Algo extraño parecía ocurrir. Pero no tan extraño si se toma en cuenta que, en 2005, con los desastres naturales ocurridos, literalmente desapareció toda la infraestructura de la red ferroviaria en Tapachula, lo que llevó al flujo a ir directamente a Arriaga, en el norte chiapaneco, y a donde el InM siguió mandando escaso personal, como si el grueso del flujo siguiera en Tapachula. Por alguna razón no se quería ver que el flujo había modificado su ruta migratoria en suelo chiapaneco.

El repunte en detenciones ${ }^{60}$ llega con el replanteamiento de la estrategia, al considerar la necesidad de detener el creciente secuestro de migrantes, específicamente en los trenes. Las atrocidades documentadas (Martínez, 2010), los testimonios de las pocas víctimas atendidas (CNDH, 2009b), la presión de las osc y los medios de comunicación, obligan al INM a cambiar su estrategia. Sólo cuando la situación empezó a ser insostenible la autoridad migratoria entendió, sin reconocer, que al haber faltado a sus obligaciones no favoreció a los migrantes, sino a la delincuencia organizada. Lo que se necesitaba no era ausentar a la autoridad, sino perfeccionar su presencia.

La administración central decide que la mejor manera de proteger a los migrantes de paso era mediante su detención y no dejándolos en manos de la delincuencia, como había ocurrido. Sin embargo, después de años de no exigirse resultados en los indicadores de detenciones, los propios agentes migratorios habían encontrado nuevas formas de adaptarse a las condiciones que le imponía la delincuencia organizada. Para 2008 la colusión de

${ }^{59}$ Esta cifra constituyó apenas 9.89 por ciento del nivel de aseguramiento del mes de marzo de 2006, que llegó a 11,435.

${ }^{60}$ En 2009 se logró asegurar a 69,033 migrantes y a 69,903 migrantes en 2010, cuando en 2005 se había llegado a la cúspide histórica de poco más de 250,000 eventos de detención. 
agentes migratorios con miembros de la delincuencia organizada, específicamente los Zetas, era un tema puntual y reiteradamente denunciado por las víctimas y organismos humanitarios.

Fuentes consultadas que prestaron sus servicios para el INM en el estado de Tabasco refieren la clara vinculación de los agentes migratorios con los Zetas para el secuestro de migrantes del tren y el manejo de casas de seguridad. Hubo empleados de bajo rango administrativo que dieron parte a la autoridad competente en su institución, por ejemplo en Tamaulipas, sin que se modificara el curso de las cosas.

2. Negociar la Ley. El debilitamiento institucional empezó con la negociación de la ley, desde los actos de corrupción de los agentes migratorios para permitir o no el paso a cambio de extorsiones hasta las negociaciones de buena voluntad con quienes hacen un trabajo humanitario a favor de los migrantes.

Con la instalación de las casas de migrantes, abiertas a instancias del acuerdo con la Pastoral de Movilidad Humana de la Iglesia católica, se inició a nivel interno - en las reuniones que el inM convocaba para sus delegadosel conflicto que se presentaba entre las disposiciones legales sobre albergue de migrantes y las posiciones de la Iglesia católica, que solicitaba que la autoridad dejara de efectuar sus funciones de verificación migratoria. El conflicto estuvo presente por años, sobre todo en San Luis Potosí, debido a la posición tildada de «legalista» de la entonces delegada de apegarse a la ley sin excepciones. ${ }^{61}$

El acuerdo INM-Iglesia católica llegó en 2007. La cercanía religiosa de la entonces titular del INM con la Iglesia católica no sólo evitó que las casas de migrantes fueran sujetas de revisión migratorias, sino que además propició que agentes de la Pastoral tuvieran una injerencia activa en los procesos de atención y desahogo de entrevistas a las probables víctimas de delitos en el interior del propio INM. Por la vía de los hechos, se inició una política pragmática de colaboración con algunos organismos civiles; como posible propó-

${ }^{61} \mathrm{La}$ funcionaria mencionada fue entrevistada un día antes de que fuera enterada de que sería removida del cargo. 
sito de transparencia en la gestión pública, no es criticable. Empero, desde un punto de vista legal, era improcedente. Faltó creatividad para iniciar una colaboración benéfica entre dependencias de gobierno e instancias civiles, se trastocó la ley, no se convocó a organismos civiles, sino que se prefirió un arreglo privado con instancias superiores de una iglesia en particular.

El acuerdo práctico que permitía que las casas de migrantes siguieran albergando migrantes sin presencia alguna de la autoridad migratoria, ${ }^{62} \mathrm{de}$ vino contrario a los propósitos que lo originaron, dado que esos espacios humanitarios fueron convertidos en trampas mortales para muchos migrantes por los delincuentes: quienes delinquen a costa de los migrantes encontraron en esos albergues y casas de migrantes espacios libres de autoridad estatal (Žižek, 2009). Testimonios de víctimas, voluntarios y responsables de esos espacios humanitarios dejan en claro que sus inmuebles no están exentas de control de la delincuencia organizada, específicamente de los Zetas, un control a contrapelo de los dirigentes de dichas casas y albergues, pero control a fin de cuentas. Miembros o colaboradores de redes delictivas han solido viajar con los migrantes en el tren (que es el medio utilizado por excelencia por los migrantes que llegan a esos espacios humanitarios), ingresan con ellos a las casas de migrantes, dentro de las mismas ubican al que guía al grupo, al que trae consigo más dinero y a los que tienen familiares en Estados Unidos. Llegada la hora de abordar el siguiente tren ocurre que, a unos metros de la casa o albergue, generalmente los migrantes son secuestrados, maltratados y los familiares extorsionados. ${ }^{63}$ Adicionalmente, volun-

62 Para el análisis de las construcciones sociales dentro de la sociedad en general, particularmente las que se distinguen por su carácter emergente, se encuentra en la obra de Niklas Luhmann. De su vasta obra se recomienda consultar Luhmann (2007a, 2007b).

${ }^{63}$ Esta realidad, además de que los migrantes lo saben, también los propios funcionarios se han dado cuenta de ello. Nótese lo que indica un delegado regional: «también se nos ha dado el caso que ahora los enganchadores son los propios connacionales de ellos, los propios centroamericanos. Entonces, qué es que va pasando [...] que llegan a veces a los albergues a la casa de caridad y empiezan a comunicarse entre ellos de qué país son, no que soy de Nicaragua, que yo también, que de dónde, que de la Ceiba; y ahí empieza la plática de ellos y entonces deciden viajar juntos para protegerse [...] toman el tren [...] y ahí sigue la amistad [...] luego, ese enganchador que es su propio connacional le dice: oye, cuando nos bajemos en Tamaulipas, conozco ahí una casa de un señor muy bueno que nos da asilo y nos da comida.

$74 \frac{\text { PRIMER SEMESTRE } 2015}{\text { MIGRACIÓN Y DESARROLLO NÚM. } 24}$ 
tarios y personal eclesial se han vuelto blanco de los delincuentes, en la medida en que defienden a los migrantes que albergan.

Desde 2008, cuando empezaron a cobrar visibilidad los casos de secuestros de migrantes. La autoridad migratoria tuvo conocimiento de que los Zetas estaban haciendo un trabajo de reclutamiento con migrantes centroamericanos, los que formarían parte de la red delictiva bajo distinta forma de adscripción, pero sólo dio parte a las autoridades judiciales, en el mejor de los casos. Ese proceder de la autoridad migratoria explica el éxito delictivo que desde esos años desarrolló la red Zetas para enganchar o secuestrar a migrantes.

3. Deficiencias en el perfil de mando. La fortaleza de una institución recae en gran medida en la solvencia ética y la capacidad profesional de quienes la conforman, una no pueda estar sobre la otra. Tradicionalmente en México la administración pública ha sido el botín repartido por parte del partido político gobernante y en el INM se ha llevado al extremo esta regla no escrita. Sería muy cuestionable que el secretario(a) de Salud fuera un ingeniero agrónomo o que el secretario de Hacienda fuera un antropólogo. Sin embargo, pareciera que a nadie sorprende que en otras posiciones quien tome la dirección de los asuntos desconozca por completo la materia.

La reflexión es pertinente porque una parte de lo ocurrido en los últimos años en relación con la migración indocumentada se encuentra relacionada con el perfil de los nombramientos de los funcionarios de alto nivel en el interior del INM y lo que ha ocurrido en la conducción de éste: su trayectoria pública no brinda elementos de juicio que permitan valorar su sapiencia en la materia. Las personas que han sido titulares de dicho instituto, todas menos una (que por cierto sólo duró unos meses en el cargo), han carecido de conocimiento previo de la materia migratoria. El o la titular ha practicado de manera regular un proceder similar con quienes conforman sus instancias subordinadas de mando a nivel central y en los estados. Antes, no existían controles de confianza y cuando se aplicaron durante la presidencia de Calderón,

Y llegando, pues es ahí donde los entrega a las bandas delictivas para su secuestro. Ese caso nos repitió en dos o tres ocasiones» (EG). 
por lo regular se encontró la manera de darles la vuelta y sólo aplicarlos a personal caído en desgracia. Ante la falta de confianza en el área interna, el último subsecretario del ramo de la administración Calderón decidió mandar al personal del INM a presentar la prueba correspondiente con el personal e instrumental del Cisen, una medida que sólo alcanzó a unos cuantos. Con la llegada de la nueva administración y la entrada de una nueva camada de funcionarios, imberbes en lo migratorio, se volvió a cambiar el procedimiento.

Por la naturaleza de la materia, el área de control y verificación migratoria hasta 2007 estuvo en manos de abogados que combinaban lo que entendían en planeación estratégica y coordinaban las acciones operativas en territorio nacional. Al igual que sus predecesores, el gobierno de Calderón en el INM designó al frente a personal con escasa experiencia para hacer frente a la complejidad del fenómeno. Las fuentes consultadas en las delegaciones señalan la pérdida de objetivos y la incapacidad para definir estrategias claras de operación. Al tiempo se hizo un nuevo nombramiento; esta vez recayó en un ingeniero en sistemas, que tampoco pudo hacer gran cosa, pues no contaba con formación y conocimiento en la materia. Es decir, la ausencia de formación y conocimiento no sólo se notaba en las instancias superiores de mando, sino también en mandos medios centrales, así como en la designación de nuevas camadas de delegados estatales que, como referente de valor para el nombramiento, contaban con la misma filiación partidaria del presidente constitucional.

En la administración pública no se puede renunciar a las obligaciones encomendadas sin que medie responsabilidad legal, como tampoco se puede negociar la ley, por nobles que sean las intenciones de quienes buscan ayudar a resolver el problema. Desde esta perspectiva, los secuestros y las masacres no sólo son el resultado de la acción de los grupos criminales: son también producto de los yerros y negligencia de las autoridades responsables de la materia migratoria que, cuando se hace evidente su errar, son enviados a otros puestos públicos sin sanción por lo equívoco de su proceder; también puede que se les sustituya con el cambio de administración o que llegue un nuevo partido al poder federal, pero igual, sin sanción por lo mal hecho o lo omitido. Ese proceder, sin fincar responsabilidad, es un valor entendido y practicado en 
el INM desde su origen hasta la fecha, al menos para sus titulares, sus directores centrales y delegados estatales.

\section{PARA Cerrar}

Por lo regular, en el análisis de las migraciones de paso en México, en particular, se omite escudriñar qué pasa en los interiores de las instituciones de gobierno que se encargan del tema migratorio. Si no se sabe de ellos, es difícil justipreciar qué hacen y qué dejan de hacer esas instituciones y su personal. En la medida en que se mantienen en la invisibilidad las limitaciones y contradicciones de la ley, los distintos problemas para su operación, la trascendencia de que se cuente o no con personal debidamente capacitado en las instancias de mando y la conjugación negativa de esos elementos, más las inercias de la burocracia y la corrupción, resulta imposible entender por qué los gravísimos daños a los migrantes de paso han llegado a ser lo que son: una constante que sigue sin ser revertida.

Lamentablemente, lo ocurrido no se debe en exclusiva a lo que pasa dentro del INM; hace falta, y eso debiera ser materia de otro análisis, investigar qué hacen y qué dejan de hacer otras instancias, como la propia Subsecretaría del ramo, la Segob, así como otras dependencias como la SRE (que por mucho tiempo ha sido contrapeso de la Segob en materia migratoria al menos), las casi invisibles secretarías del Trabajo y Previsión Social, de Educación Pública, Desarrollo Social y Economía, que durante decenios han estado en ayunas de asumir su responsabilidad institucional en materia migratoria, así como el Sistema de Seguridad Nacional y de lucha contra el crimen organizado, que han hecho particular hincapié en sus prioridades sobre las cuestiones migratorias, etcétera. Es decir, existe, sólo desde esta breve señalización, un conjunto de instituciones de gobierno que participan, por mandato de ley, de lo migratorio y que no han sido debidamente analizadas. Desde este amplio horizonte, lo argumentado es, como dice el título, «Notas...», que podrán tener mejor dimensión cuando se cuente con esos análisis mencionados y hoy ausentes. 
En México, en abundancia de referentes necesarios, las deficiencias en la actuación institucional y sus problemas organizativos, de operación y coordinación interinstitucional, conjugados con la extensa colaboración de autoridades locales, municipales, estatales y federales con grupos criminales, y el hecho de que por otra parte, se carece de un sistema adecuado y eficiente para atender a las víctimas migrantes,${ }^{64}$ dan por resultado una permanente violación a los derechos humanos de los mismos migrantes. En este sentido, es importante analizar los efectos de las políticas migratorias gubernamentales, sin duda, pero es igualmente importante conocer cómo se gestan esas políticas, quiénes son los actores intra-institucionales que formulan, diseñan y ordenan que esas políticas se apliquen. De otra forma, las propuestas de mejora de política pública pueden ser presentadas o canalizadas a instancias equívocas. En el caso de México, son varias las instancias federales que tienen que ver con lo migratorio, como se ha dicho un poco antes, aunque en este texto se ha dado, por razones de espacio y necesidad de abundar en lo específico de la dependencia, atención prioritaria al acontecer interno del INM. En su momento, habrá que analizar el papel formal y real de esas otras instancias de gobierno, hoy omisas no sólo en la actuación, sino en los análisis sobre la actuación gubernamental en materia migratoria internacional.

La violación a los derechos humanos, por acción o por omisión, de parte de las instancias gubernamentales, se conjugan con los abusos y violencias de distinta índole que ejercen miembros de las comunidades por donde transitan los(as) migrantes y, ciertamente, por parte de los grupos dedicados al crimen

${ }^{64}$ Las entrevistas indican que, en caso de que el migrante sea víctima de algún delito, no es función propia de la autoridad migratoria hacer el procedimiento correspondiente, sino tan sólo remitirlos a las instancias convenientes para hacer su denuncia (entrevistas con LA, EG, GL y RR). Para poner un ejemplo del procedimiento: «Nosotros lo que hacemos es el acompañamiento jurídico, le tomamos su declaración, no con preguntas, la declaración que ellos nos hacen y le damos la opción al migrante, porque eso es un tema de derechos humanos, que nosotros no podemos presionarles y decirles: tú tienes que ir a denunciar, tienes la posibilidad de denuncia, se les dice sus derechos; tú eres víctima de un delito, es importante que denuncies, quieres hacerlo. Sí quiero hacerlo. Entonces vamos y lo presentamos ante la PGR, si es delito de fuero federal y si es una cuestión de fuero común ante la PGJE. Se presenta, se le toma toda su declaración y se inician las investigaciones correspondientes a partir de la autoridad ministerial» (AM). 
(Keane, 2000; Labica, 2008; Sanmartín, 2004). Es decir, la violencia social, en sus infinitos vestidos sociales, es otra vertiente por investigar. Sin ella, cualquier análisis es parcial, aunque sea difícil elaborarlo, como es el caso del presente escrito que se centró en el INM.

\section{Bibliografía}

CASILLAS R., Rodolfo (2011), "La labor humanitaria y los organismos civiles: la experiencia de los albergues y casas de migrantes, realidades y desafíos", en Leonir M. Chiarello (coord.), Las políticas públicas sobre migraciones y la sociedad civil en América Latina. Los casos de Argentina, Brasil, Colombia y México, Nueva York, Scalabrini International Migration Network.

(2012), "La construcción del dato oficial y la realidad institucional: la disminución del flujo indocumentado en los registros del InM», Migración y Desarrollo, vol. 10, núm. 19.

Celorio, Mariana (2014), "Organizaciones emergentes en torno a migración en tránsito por México-EUA: solidarias y perversas», en Alejandro Méndez y Silvia Molina (coords.), Comunicación, transmigración y sistemas emergentes, vol. I, México, UNAM.

Comisión Nacional de los Derechos Humanos (CNDH) (2009a), Informe especial de la Comisión Nacional de los Derechos Humanos sobre el caso de secuestro en contra de migrantes, México, 15 de junio.

(2009b), Bienvenidos al infierno del secuestro. Testimonios de migrantes, México, CNDH.

Keane, John (2000), Reflexiones sobre la violencia, Madrid, Alianza.

LabiCA, Georges (2008), «Para una teoría de la violencia», Polis, núm. 19.

Luhmann, Niklas (2007a), La sociedad de la sociedad, México, Herder. (2007b), Sociología del riesgo, México, Universidad Iberoamericana.

MARTínez, Óscar (2010), Los migrantes que no importan, México, Sur.

Ruggiero, Vincenzo (2009), La violencia política: un análisis criminológico, Barcelona, Anthropos/Universidad Autónoma Metropolitana.

Sanmartín, José (coord.) (2004), El laberinto de la violencia. Causas, tipos y efectos, Barcelona, Ariel. 
RODOLFO CASILLAS R.

et al. (coords.) (2010), Reflexiones sobre la violencia, México, Siglo XXI Editores/ICRS.

SeGATo, Rita Laura (2003), Las estructuras elementales de la violencia; ensayos sobre género entre la antropología, el psicoanálisis y los derechos humanos, Buenos Aires, Universidad Nacional de Quilmes.

SoFskY, Wolfgang (2004), Tiempos de horror. Amok, violencia, guerra, Madrid, Siglo XXI Editores.

(2006), Tratado sobre la violencia, Madrid, Abada.

StRAnge, Susan (2003), La retirada del Estado; la difusión del poder en la economía mundial, Barcelona, Icaria/Intermón Oxfam.

WaCoUAnt, Loïc (2010), Castigar a los pobres. El gobierno neoliberal de la inseguridad social, Barcelona, Gedisa.

ŽIŽEK, Slavoj (2009), Sobre la violencia. Seis reflexiones marginales, Barcelona, Paidós.

Zúniga, Leonor (2010), "La soledad de los migrantes», Perspectivas, núm. 43. 